\title{
Micromagnetic Simulation of Antiferromagnetic/Ferromagnetic Structures
}

\author{
D. Suess, T. Schrefl, W. Scholz, J.-V. Kim, R. L. Stamps, and J. Fidler
}

\begin{abstract}
A novel approach for solving the Landau-Lifshitz-Gilbert equation for antiferromagnets with the finite-element method is presented. The antiferromagnet is treated in a continuum theory which allows us to explore the domain structure on a mesoscopic length scale. The finite-element method is suitable to treat antiferromagnets with arbitrarily shaped grains as well as exchange coupled antiferromagnetic (AF)/ferromagnetic (F) structures. The change of the domain configuration in the antiferromagnet after the reversal of the ferromagnet leads to exchange bias in AF/F bilayers with perfectly compensated interfaces.
\end{abstract}

Index Terms-Antiferromagnetic (AF) domain structure, antiferromagnets, exchange bias, finite-element method, micromagnetics.

\section{INTRODUCTION}

$\mathbf{R}$ ECENT theoretical models of antiferromagnetic (AF)/ferromagnetic $(F)$ interfaces start from a discrete lattice of spins [1]. Here we present finite element simulations based on a continuum model to treat magnetization processes in antiferromagnets. This novel technique allows us to take into account structural features like polycrystalline grains and resolves AF domain structures at a mesoscopic lengthscale. We focus on perfectly compensated interfaces, where there is an equal number of positive and negative exchange interactions across the interface. Koon [2] demonstrated that the F magnetization will align perpendicular to the AF magnetization if the interface is fully compensated. This so-called spin flop coupling is attributed to the frustration of the interface spins at the compensated interface.

Section II of this paper presents a continuum approach to treat magnetization processes of antiferromagnets. Then we verify the novel algorithm comparing the results with atomistic calculations obtained for a AF/F. Section III shows results for an $\mathrm{AF} / \mathrm{F}$ bilayer consisting of a $\mathrm{F}$ thin film on top of a polycrystalline antiferromagnet.

\section{CONTINUUM THEORY AND FEM}

In F materials, spins do not change significantly from lattice point to lattice point owing to $\mathrm{F}$ exchange coupling. In $\mathrm{AF}$ materials the direction of the spins usually changes its directions

Manuscript received February, 14, 2002; revised May 28, 2002. This work was supported in part by the Austrian Science Fund under Grant Y 132 PHY and in part by the Australian Research Council.

D. Suess, T. Schrefl, W. Scholz, and J. Fidler are with the Vienna University of Technology, A-1040 Vienna, Austria (e-mail: dieter.suess@tuwien.ac.at).

J.-V. Kim and R. L. Stamps are with The University of Western Australia, Crawley WA 6009, Australia.

Digital Object Identifier 10.1109/TMAG.2002.803594.

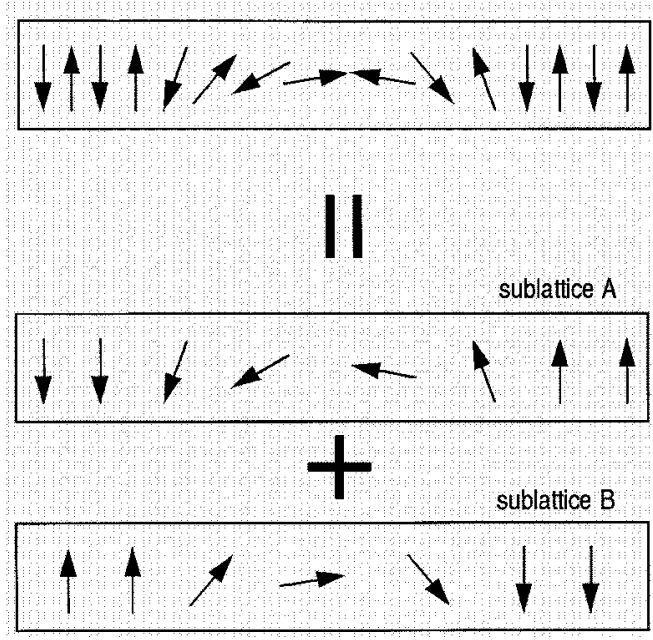

Fig. 1. Schematic diagram that shows the subdivision of an antiferromagnet into two sublattice. The AF wall structure can be represented in a continuum model by two walls, one in each sublattice.

from lattice point to lattice point, which would result in a discretization length equal to the atomic lattice constant. However, if the antiferromagnet is subdivided into sublattices, again the magnetic moments varies slowly in space within each sublattice (Fig. 1).

We start from the Heisenberg's exchange energy to find the exchange energy in continuum limit. When the spin operators are approximated as classical vectors the exchange energy for $N$ spins can be written as

$$
E_{\mathrm{ex}}=-\sum_{i=1}^{N} \sum_{j \neq i}^{N} J_{i j} \mathbf{S}_{i} \mathbf{S}_{j}
$$

where $J_{i j}$ is the exchange integral. After the subdivision into two sublattices it follows

$$
E_{\mathrm{ex}}=\left(-J \sum_{i=1}^{N / 2} \mathbf{S}_{i}^{A} \sum_{j}^{n N} \mathbf{S}_{j}^{B}\right)+\left(-J \sum_{i=1}^{N / 2} \mathbf{S}_{i}^{B} \sum_{j}^{n N} \mathbf{S}_{j}^{A}\right)
$$

where $n N$ denotes the number of nearest neighbors. In a continuum limit, we want all our variables to be evaluated at the same point. Neighboring spins of one sublattice are indirectly coupled to each other, as spins $\mathbf{S}_{i}^{A}$ acts on $\mathbf{S}_{i}^{B}$ and $\mathbf{S}_{i}^{B}$ acts on $\mathbf{S}_{i+1}^{A}$. The sum over nearest neighbors is expanded about $\mathbf{x}_{i}$

$$
E_{\mathrm{ex}}=-2 J \sum_{i=1}^{N / 2} \mathbf{S}^{A}\left(\mathbf{x}_{i}\right) \sum_{k=1}^{n N} \mathbf{S}^{B}\left(\mathbf{x}_{i}+\delta_{k}\right) .
$$


If the position of the spins form a cubic lattice (lattice constant $=a), \delta_{k}$ points to the six neighbors along the cube edges. Linear terms cancel because of the cubic symmetry. Thus, we get

$$
\sum_{k=1}^{6} \mathbf{S}^{B}\left(\mathrm{x}_{i}+\delta_{k}\right)=6 \mathbf{S}^{B}\left(\mathrm{x}_{i}\right)+a^{2} \Delta \mathbf{S}^{B}\left(\mathrm{x}_{i}\right) .
$$

After replacing the sum in (3) by an integral and applying First Greens Identity with the condition that the normal derivative of the magnetic polarization at the surface vanishes, we get for the exchange energy

$$
\begin{aligned}
E_{\mathrm{ex}}=-\frac{6 J S^{2}}{a^{3}} \frac{1}{2} \int_{V} \mathbf{u}^{A}\left(\mathbf{x}_{i}\right) \mathbf{u}^{B}\left(\mathbf{x}_{i}\right) d V \\
+\frac{J S^{2}}{a} \int_{V}\left(\sum_{k=x, y, z} \nabla u_{k}^{A}\left(\mathbf{x}_{i}\right) \nabla u_{k}^{B}\left(\mathbf{x}_{i}\right)\right) d V
\end{aligned}
$$

where $\mathbf{u}^{A}\left(\mathbf{x}_{i}\right)=\mathbf{S}^{A}\left(\mathbf{x}_{i}\right) / S$ and $\mathbf{u}^{B}\left(\mathbf{x}_{i}\right)$ denote the directions of the spontaneous polarization for sublattices $A$ and $B$, respectively. In analogy to ferromagnets the normal derivative of the magnetization vanishes at a free surface. The normal derivative of the magnetization at the AF/F interface is neglected. Antiphase boundaries can be included by locally changing the exchange integral.

For the anisotropy energy, Zeeman energy and stray field the continuum expressions are the same as for ferromagnets. These three energy contributions have to be calculated for both sublattices. The stray field obtained in a continuum model does not take care of the atomic arrangement of the magnetic dipoles. The corresponding field term has the form of an anisotropy field. Thus, it is usually included in the anisotropy constant.

At a interface between a ferromagnet and an antiferromagnet we calculated the interface energy as

$$
\begin{aligned}
E_{\text {ex }, \text { interface }}=-2 J \sum_{i=1}^{I_{A}} \mathbf{u}^{A, F}\left(\mathbf{x}_{i}\right) \mathbf{u}^{B}\left(\mathbf{x}_{i}\right) & \\
& -2 J \sum_{i=1}^{I_{B}} \mathbf{u}^{B, F}\left(\mathbf{x}_{i}\right) \mathbf{u}^{A}\left(\mathbf{x}_{i}\right)
\end{aligned}
$$

where $\mathbf{u}^{B, F}\left(\mathbf{x}_{i}\right)$ and $\mathbf{u}^{A, F}\left(\mathbf{x}_{i}\right)$, denote the directions of the spontaneous polarization in the ferromagnet for sublattice $A$ and $B$, respectively. $I_{A}$ and $I_{B}$ are the number of $A$ and $B$ sites at the interface.

In principle the polarization of sublattices $A$ and $B$ point parallel in the bulk of the ferromagnet. Thus, one sublattice yields redundant information. However, on the interface to the antiferromagnet the spins of the ferromagnet may be canted. Hence, both sublattices are required.

The time evolution of the magnetic polarization is calculated using the Landau-Lifshitz-Gilbert (LLG) equation.

In order to test the micromagnetic approach, we compared the finite element calculations with an atomistic approach for Koon's model of an exchange coupled ferromagnet/antiferromagnet bilayer. In order to observe domain walls in the antiferromagnet which are wound up past $90^{\circ}$, it is required to introduce an in-plane anisotropy.

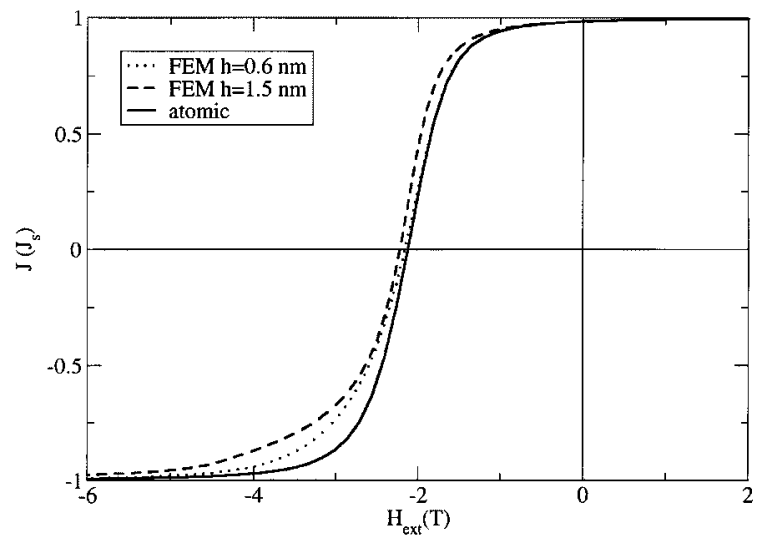

Fig. 2. Comparison of the hysteresis loop for different mesh sizes with an atomistic calculation.

The thickness of the F and the AF layer is $2 \mathrm{~nm}$ and $10 \mathrm{~nm}$, respectively. The interface between the ferromagnet and the antiferromagnet, which is parallel to the $x-y$ plane, is perfectly compensated. In the atomistic approach the ferromagnet/antiferromagnet is modeled as a simple cubic structure. The total energy is calculated using a Heisenberg Hamiltonian. Details of the atomistic approach can be found in [3].

A uniaxial anisotropy along the $y$ axis, with $K_{1}^{A}=K_{1}^{B}=$ $5.45 \times 10^{5} \mathrm{~J} / \mathrm{m}^{3}$, is assumed for the antiferromagnet. The exchange constant is $A=-2.725 \times 10^{-12} \mathrm{~J} / \mathrm{m}$, and $J_{s}^{A}=J_{s}^{B}=$ $0.5 \mathrm{~T}$.

No uniaxial anisotropy is assumed in the ferromagnet. The exchange constant in the ferromagnet is $A=3.6 \times 10^{-11} \mathrm{~J} / \mathrm{m}$ and $J_{s}=1 \mathrm{~T}$. Additionally an in plane anisotropy which favors spin rotation in the $x-y$ plane is assumed in the ferromagnet and antiferromagnet $\left(K=8 \times 10^{7} \mathrm{~J} / \mathrm{m}^{3}\right)$. The exchange constant across the interface is $A=-8.17 \times 10^{-12} \mathrm{~J} / \mathrm{m}$.

Linear basis functions are used to interpolate the magnetization. Tetrahedrons form the finite element mesh. The calculations were performed for different average edge length of one tetrahedron $(1.5,1.0$, and $0.6 \mathrm{~nm})$. The external field is applied $10^{\circ}$ off the $x$ axis in the $x-y$ plane.

Fig. 2 compares the hysteresis loops of the finite element calculation with the atomistic model. With decreasing mesh size the finite element calculation converges toward the atomistic method. Similar convergence can be seen in Fig. 3, which shows the profile of the domain wall, which is formed in the antiferromagnet during reversal of the ferromagnet. The $x$ component of the magnetic polarization is plotted as a function of the distance of the interface. The discontinuity at position 0 occurs owing to the $90^{\circ}$ coupling between the ferromagnet and antiferromagnet. Also, the canted state of the interface spins (I) of the AF is resolved by the finite-element method.

\section{IrMn/Permalloy Bilayer}

The continuum model is suitable to calculate antiferromagnets with large domain wall width such as IrMn. It forms a disordered FCC $(\gamma)$ phase [4]. We treat the system with a two lattice approach and assume the following intrinsic parameters: $A=-10^{-11} \mathrm{~J} / \mathrm{m}, K_{1}^{A}=K_{1}^{B}=10^{\check{5}} \mathrm{~J} / \mathrm{m}^{3}, J_{s}^{A}=J_{s}^{B}=0.5 \mathrm{~T}$ and lattice constant $a=3.76 \times 10^{-10} \mathrm{~m}$. For permalloy $K_{1}=$ 


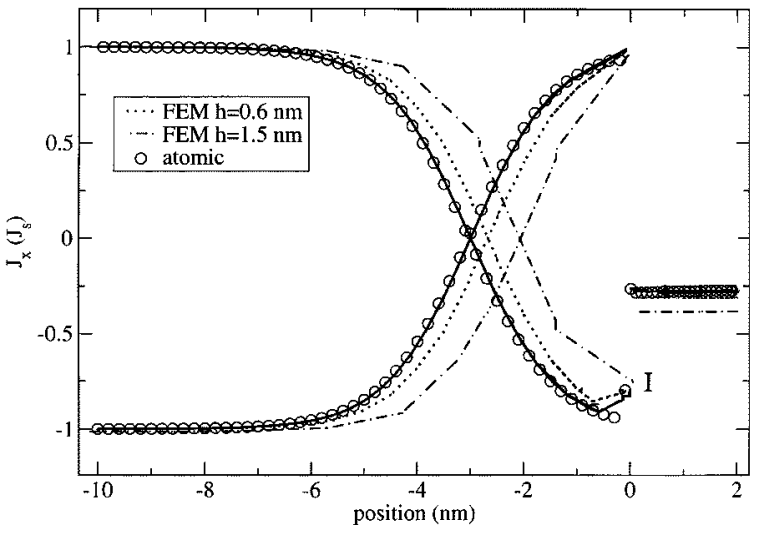

Fig. 3. Under the action of an applied field a domain wall forms in the antiferromagnet. The curves compare the domain wall profile of finite element calculations with the atomistic calculation. At point I the spins at the surface of the $\mathrm{AF}$ are canted and are no longer antiparallel to each other.

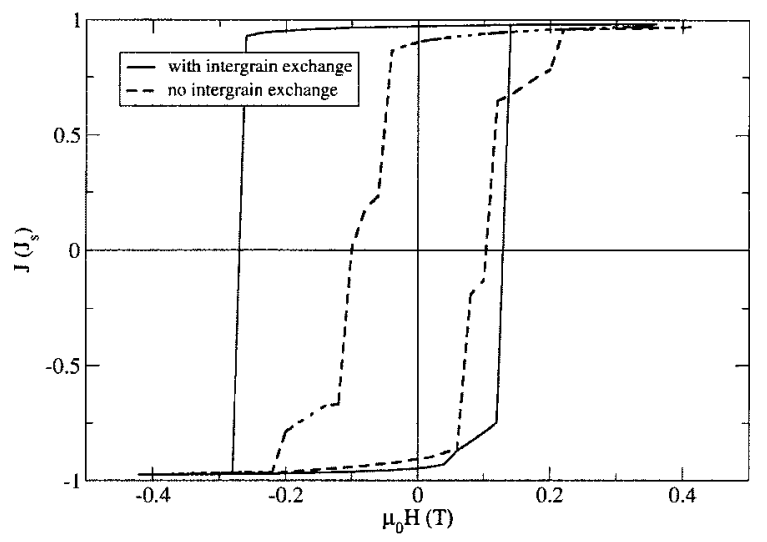

Fig. 4. Hysteresis loops of a ferromagnet and an F/AF bilayer with compensated interface. The AF leads to an enhanced coercivity and to a loop shift.

$2 \times 10^{3} \mathrm{~J} / \mathrm{m}^{3}, A=10^{-11} \mathrm{~J} / \mathrm{m}, J_{s}=1 \mathrm{~T}$. The easy axis of the permalloy is along the $x$ axis. No in-plane anisotropy is assumed in the ferromagnet and in the antiferromagnet. The thickness of the permalloy layer is $2 \mathrm{~nm}$ and of the IrMn layer $6 \mathrm{~nm}$. The IrMn layer consists of $8 \times 3$ grains. The grain size is $8 \mathrm{~nm}$. The basal planes of the grains are squares, equal in size. The easy axes are randomly oriented in space. The AF grains are weakly exchange coupled to each other. We assume an intergranular phase with a thickness of $1 \mathrm{~nm}$ with reduced exchange constant, $A=5 \times 10^{-13} \mathrm{~J} / \mathrm{m}$ or no exchange. The interface between the permalloy layer and the IrMn layer is perfectly compensated.

The solid line of Fig. 4 gives the hysteresis loop for weak exchange between the AF grains. The loop shows bias, although

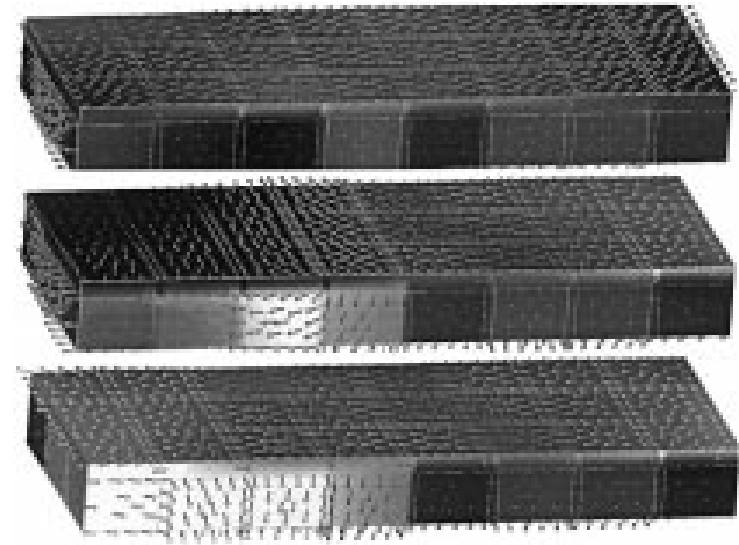

Fig. 5. Nonequilibrium states during switching of a IrMn/Permalloy bilayer. The antiferromagnet (AF) consists of $8 \times 3$ grains. The size of each grain is $8 \times 8 \times 6 \mathrm{~nm}^{3}$. Each grain is subdivided into about 400 finite elements.

the interface is completely compensated. In addition Fig. 4 gives the hysteresis loop for zero exchange between the AF grains. In this case no bias occurs. These results indicate that a weak intergrain exchange interaction is required to obtain exchange bias.

Fig. 5 shows a sequence of magnetization configurations during the reversal of the ferromagnet for weak exchange between the AF grains. From top to bottom the magnetization of the ferromagnet reverses its orientation. Initially the magnetization of the AF grains in the front point in the same direction. When the ferromagnet is reversed the $\mathrm{AF}$ grains on the left-hand side switch, whereas the AF grains on the right-hand side remain in their original direction. As a consequence, a domain wall is formed in the antiferromagnet. The increase of the domain wall energy results in exchange bias.

The presented results show that the increase in domain wall energy contributes to exchange bias. To observe a change of the $\mathrm{AF}$ domain configuration it is crucial that some and not all $\mathrm{AF}$ grains switch irreversibly as the ferromagnet is reversed.

\section{REFERENCES}

[1] T. C. Schulthess and W. H. Butler, "Consequences of spin-flop coupling in exchange biased films," Phys. Rev. Lett., vol. 81, pp. 4516-4519, 1998.

[2] N. C. Koon, "Calculations of exchange bias in thin films with F/AF interfaces," Phys. Rev. Lett., vol. 78, pp. 4865-4868, 1997.

[3] J.-V. Kim, R. L. Stamps, B. V. McGrath, and R. E. Camley, "Angular dependence and interfacial roughness in exchange-biased F/AF bilayers," Phys. Rev. B, vol. 61, p. 8888, 2000.

[4] A. E. Berkowitz and K. Takano, "Exchange anisotropy-A review," J. Magn. Magn. Mater., vol. 200, pp. 552-570, 1999. 